\title{
LA TEORIA DELLA FOTOIONIZZAZIONE DI CHAPMAN IN ATMOSFERA NON ISOTERMA (*)
}

\author{
F. MARIANI
}

Nello studio della ionizzazione negli strati alti dell'atmosfera, cioè nella zona in cui è localizzata la ionosfera, si considera generalmente come teoria fondamentale quella della fotoionizzazione formulata da Chapman ( $\left.{ }^{1}\right)\left({ }^{2}\right)$.

Tale teoria fu principalmente applicata al caso della formazione dello strato ionosferico che si forma intorno ai $100 \mathrm{~km}$ dal suolo, strato $E$ normale: in verità lo spessore dello strato $E$ è assai piccolo, pochi km, e percio l'ipotesi della costanza della temperatura che la teoria esplicitamente presuppone, almeno nell'intervallo di quote in cui praticamente si manifesta la fotoionizzazione, si può ritenere sufficientemente giustificata. In seguito la teoria di Chapman è stata applicata senza modificazioni anche allo studio degli strati più alti, strati $F_{1}$ e $F$, il cui spessore è abhastanza grande perché, a priori, lipotesi della costanza della temperatura nelle regioni interessate possa ritenersi senz'altro valida. D'altra parte numerose osservazioni sperimentali e considerazioni teoriche indicano una reale e sensibile variazione di temperatura con la quota anche se poi numericamente gli andamenti di essa o $i$ singoli valori misurati risultano anche assai diversi per i vari autori (3): sembra provato che la temperatura, almeno fino ad una certa quota, debha crescere più o meno rapidamente; a quote superiori essa può però anche assumere un valore costante ovvero tornare a decrescere.

Gledhill e Szendrei (t) hanno studiato il caso in cui la temperatura dell'atmosfera cresce linearmente e indefinitamente con l'altezza, considerando la terra di raggio infinito.

Nella presente nota si dà una formulazione della teoria di Chapman nella quale la temperatura della regione nella quale si manifesta la ionizzazione viene supposta variabile con legge arbitraria; tale legge viene poi precisata come segue: andamento lineare di tem-

(*) Comtunicazione presentata alla «Association Internationale di Magnetisme et electricité terrestre » nella X Assemblea Generale dell'U.G.G.I. - Roma 195t. 
peratura fino a una quota $c$, costante a quote magriori; il calcolo viene effettuato per $i$ due casi di stratificazione piana e di stratificazione sferica dell'atmosfera, rispettivamente corrispondenti al considerare la Terra con ragrio infinito oppure finito. Si confrontano infine e si discutono più in dettaglio $i$ risultati analitici ottenuti.

1) Caso della stratificazione piana. - Sia $T(z)=T_{0} f(z)$ la legrge di variazione della temperatura assoluta $T$ con la quota $z$, essendo $T_{0}$ la temperatura alla quota $z=0$; orientiamo l'asse $z$ positivamente verso l'alto; la $f(z)$ e per il momento lasciata arbitraria e soddisfa lunica condizione $f(0)=1$.

L'andamento della densita di materia p con la quota is dato dalla equazione

$$
\frac{d}{\rho} \underline{\rho}=-\left[\frac{1}{H_{\mathrm{o}}}+\frac{d f}{d z}\right] \frac{d z}{f(z)} \text { con } H_{\mathrm{s}}=\frac{k T_{\mathrm{o}}}{m g}
$$

che, in termini finiti, dà

$$
p=p_{o} \exp \left[-\frac{1}{H_{\mathrm{o}}} \int_{0}^{\mathrm{z}}\left(1+H_{-} \frac{d f}{d z}\right) \frac{d z}{f(z)}\right]
$$

con $\rho_{o}=\rho(0), k$ costante di Boltzmann, $m$ massa molecolare media, $g$ accelerazione di gravità.

Sia $S(z)$ l'intensità della radiazione ionizzante alla generica quota z; l'assorbimento è allora descritto dalla relazione differenziale

$$
\frac{d S}{S}=\frac{A_{0}}{[f(z)]^{\mathrm{n}-1}} \rho(z) \sec \chi d z-h \sec \chi \varphi(z) d z
$$

$\operatorname{con} \quad h=A_{\mathrm{o}} \rho_{\mathrm{o}} ; \varphi(z)=\exp \left[-\frac{1}{H_{\mathrm{o}}} \int_{\mathrm{o}}^{\mathrm{z}}\left(1+H_{\mathrm{o}} \frac{d f}{d z}\right) \frac{d z}{f(z)}\right] \cdot[f(z)]^{-\mathrm{n}+-1}$

ove il coefficiente di assorbimento $\frac{A_{0}}{[f(z)]^{n-1}}$ è però stato assunto funzione di $z\left(\operatorname{con} n\right.$ per il momento arbitrario) e di valore $A_{0}$ alla quota $z=0$.

Si deduce dalla [3]

$$
S(z)=S^{*} \exp [h \sec \chi g(z)] \operatorname{con} g(z)=\int_{c}^{z} \varphi(\xi) d \xi
$$


ove $c$ è la quota alla quale la temperatura assume un determinato valore $T_{1}$ e $S^{*}=S(c)$ è funzione di $\%$, angolo zenitale del Sole (ma non di $z$ come invece sarà nel caso di raggio terrestre finito).

II numero di ioni prodotti per $\mathrm{cm}^{3}$ e per sec si scrive, $\operatorname{con} \beta$ costante di proporzionaliti,

$$
\begin{aligned}
I(z)=\beta \cos \chi \frac{d S}{d z} & =\beta h S^{*} \exp [h \sec \% g(z)+ \\
& \left.-\frac{1}{H_{0}} \int_{0}^{z}\left(1+H_{0} \frac{d f}{f(z)}\right) \frac{d z}{f(z)}\right] \cdot[f(z)]^{-\mathrm{n}+1}
\end{aligned}
$$

Valori estremi relativi di $I(z)$ si hanno eventualmente per quei valori di $z$ che soddisfano l'equazione $\frac{d I}{d z}=0$ che si traduce nell'altra

$$
h s \in c \% \frac{d g}{d z}-\frac{1}{H_{o}}\left(1+H_{\mathrm{o}} \frac{d f}{d z}\right) \frac{1}{f^{\prime}(z)}-\frac{n-1}{f(z)} \frac{d f}{d z}=0
$$

ovvero, indicando con $\bar{z}$ il generico valore di $z$ che la soddisfa:

$$
\text { h sec } \% \exp \left[-\frac{1}{H_{\mathrm{o}}} \int_{\mathrm{o}}^{\overline{\mathrm{z}}}\left(1+H_{0}^{-} \frac{d f}{d z}\right) \frac{d z}{f(z)}\right][f(\bar{z})]^{-n+2}-\left[\frac{1}{H_{\mathrm{o}}}+n\left(\frac{d f}{d: z}\right)\right]=0
$$

La [7] è formula di validità del tutto generale quale che sia l'andamento di $f(z)$; per procedere introdurremo la ipotesi che la temperatura sia funzione lineare di $z$. Porremo pertanto $f(z)=1 ; p z$ con le due possiljilità $p>0$ e $p<0$ corrispondenti ad un aumento o ad una diminuzione della temperatura con la quota. La [7] diviene in questo caso

$$
h \sec \chi(1+p \bar{z})^{x}-\left(n p+\frac{1}{H_{\mathrm{o}}}\right)=0 \text { con } r-1-n-\frac{1}{H^{H} H_{.}}
$$

da cui si deduce

$$
\frac{T(\bar{z})}{T_{\mathrm{o}}}=\frac{\bar{T}}{T_{0}}=1+p \bar{z}=\frac{T^{*}}{T_{\mathrm{o}}} \sec ^{-1 / \mathrm{r}} \chi
$$

con

$$
\frac{T^{*}}{T_{n}}=\left(\frac{n p+\frac{1}{H_{\mathrm{o}}}}{h}\right)^{1 / \mathrm{r}}
$$


ove $T^{*} \grave{e ̀ ~ l a ~ t e m p e r a t u r a ~ c h e ~ s i ~ h a ~ a l l a ~ q u o t a ~ a l l a ~ q u a l e ~} I(z)$ è massima quando il Sole è esattamente allo zenith. Si osservi che, perché al crescere di $\chi \ell$ la quota del massimo di $I(z)$ cresca, come sembra suggerito dall'esperienza, deve risultare $r<0$ per $p>0$ e $r>0$ per $p<0$.

La [5] diviene, nel caso particolare che stiamo considerando,

$$
I(z)=\beta h S^{*} \operatorname{ex} p\left\{\frac{h s^{\prime} c \chi}{p r}\left[(1+p z)^{\mathrm{r}}-(1+p c)^{\mathrm{r}}\right]\right\} \cdot(1+p z)^{r-1}
$$

Si nota che, ponendo $c=\infty$ e $S^{*}=S_{\infty}$, si riottengono i risultati di Gledhill e Szendrei.

Il valore massimo di $I(z)$ per la [9] dipende da $\chi$ : si ha precisamente $\left({ }^{\star}\right)$.

$$
I_{\mathrm{MZ}}=I(\bar{z})=\beta h \mathrm{~S}^{*}+x p\left\{\frac{h \sec \chi}{p \boldsymbol{r}}\left[\left(\frac{T}{T_{\mathrm{c}}}\right)^{\mathrm{r}}-\left(\frac{T_{1}}{T_{\mathrm{r}}}\right)^{\mathrm{r}}\right]\right\} \cdot\left(\frac{T}{T_{\mathrm{o}}}\right)^{\mathrm{r}-1}
$$

ovvero anche

$$
\begin{gathered}
I_{\mathrm{MZ}}=\beta h S^{*}\left(\frac{T^{*}}{T_{\mathrm{o}}}\right)^{\mathrm{r}-1} \exp \left[\frac{1+n p H_{\mathrm{o}}}{1-(\mathrm{i}-n) p H_{\mathrm{o}}}\left(\mathrm{s}^{\mathrm{r}} \sec \chi-1+\ln \cos \gamma\right)\right], \\
\operatorname{con} s=\frac{T_{!}}{T^{*}} ;
\end{gathered}
$$

ove si ammettesse $S^{*}=S_{\infty}$ indipendente da $\chi$ e uguale a $S(\infty)$, la [12] diverrebbe

$$
I_{\mathrm{MZ}}=I_{\mathrm{o}} \exp \left\{\frac{1+n \cdot H_{\mathrm{o}}}{1-(1-n) p H_{\mathrm{o}}}\left[\mathrm{s}^{\mathrm{r}}(\operatorname{ser} \%-1)+\ln \cos \%\right]\right\}
$$

con

$I_{\mathrm{o}}=\beta h S_{\circ}\left(\frac{T^{*}}{T_{\mathrm{o}}}\right)^{\mathrm{r}-1} \exp \left|\frac{1+n p H_{\mathrm{o}}}{1-(1-n) p H_{\mathrm{o}}}\left(s^{\mathrm{r}}-1\right)\right|=I_{\mathrm{MZ}}(\chi=0)\left[13^{\prime}\right]$

Per determinare la dipendenza di $S^{*}$ da $\%$ ammettiamo che la temperatura al disopra della quota $z=c$ assuma un valore costante

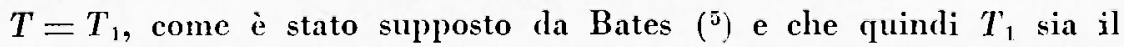
valore della temperatura nell'intervallo di quote $c-100$.

(*) Qui e nel seguito conveniamo di indicare con,$(x)$ il massimo di una fan. zione $y=y(z . \chi)$ rispetto alla variabile $z$ e con $y_{\text {u }}$ il massimo della funzione $y_{M Z}(\%)$ rispetto a $\chi$. 
Con una analisi analoga a quella che ci ha condotto alla [4] si stabilisce anche per $z>c$ l'andamento della intensità della radiazione ionizzante $S(z)$ e della intensità di ionizzazione che, per distinguerla dalla $I(z)$ definita nell'intervallo $0 \leqslant z \leqslant c$, indicheremo con il simbolo $J(z)$ : si ha precisamente

$$
\begin{gathered}
S(z)=S_{\infty} \exp \left[-\alpha \sec \psi \cdot \exp \left(-\frac{z-c}{\psi H_{\mathrm{o}}}\right)\right] \\
\operatorname{con} \psi=\frac{T_{1}}{T_{\mathrm{o}}}=1+p c \text { e con } \alpha=\left(1+n p H_{\mathrm{o}}\right) \mathrm{s}^{\mathrm{r}} .
\end{gathered}
$$

In quanto a $J(z)$ si ha

$$
J(z)=J(c) \exp \left\{\alpha \sec \gamma\left[1-\exp \left(-\frac{z-c}{\downarrow H_{\mathrm{o}}}\right)\right]-\frac{z-c}{\downarrow H_{\mathrm{o}}}\right\}
$$

con

$$
J(c)=\frac{\beta \alpha S_{\infty}}{\psi H_{0}} \exp [-\alpha \sec \chi] .
$$

Si constata che la $J(z)$ definita dalla [15] ha un eventuale massimo relativo per $z$ tale che

$$
\exp \left[-\frac{\bar{z}-c}{\dot{\psi} H_{\mathrm{o}}}\right]-\frac{\cos \psi}{\alpha}=0
$$

Perché il valore di $z$ soddisfacente tale equazione sia $\geqslant c$ deve perì risultare $\frac{\cos \gamma}{\%}<1$. Il valore di $J(z)$ in corrispondenza di $\bar{z}$ diviene, per la $[16\rceil$,

$$
J(\bar{z})=J_{\mathrm{MZ}}=J(c) \frac{\operatorname{ccs} \gamma}{\alpha} \exp [\alpha \sec \chi-1]
$$

che, tenendo conto della $\left[15^{\prime}\right]$, diviene ancora

$$
J_{\mathrm{MZ}}=\frac{\beta S_{\infty}}{\downarrow H_{\mathrm{o}}} \cos \% \exp [-1]
$$

cosicché si può porre la $\left[15^{\prime}\right]$ nella forma

$$
J(c)=J_{\mathrm{Mz}} \alpha \text { sec } \chi \exp [1-x \sec \chi] .
$$

Il valore del massimo $J_{\text {yy }}$ si otticne allora dalla [18] che mostra essere $J_{M z}$ una funzione monotona decrescente al crescere di $\chi$; quin- 
di il valore di $J_{\ldots,}$ si consegue allorché $\cos \chi$ ha il massimo valore consentito dalla [16] stessa, ciò̀ $\alpha$, se è $\alpha \leqslant 1$ e l se $\alpha>1$, per cui scriviamo senz'altro

$$
J_{M \chi}=\left\{\begin{array}{l}
\frac{\beta \infty}{\psi H_{0}} \alpha \exp [-1] \quad \text { se } \alpha<1 \\
\frac{\beta \infty}{\psi H_{0}}, x p[-i] \quad \text { se } \alpha>1 .
\end{array}\right.
$$

Ritorniamo ora alla [12]: i risultati fin qui ottenuti ci consentono di scrivere la [12] stessa, tenendo conto della dipendenza di $S^{*}$ da $\chi$ fornita dalla [14], nella forma

$$
I_{\mathrm{sZ}}=I_{\mathrm{o}} \exp [F(\chi)]
$$

ove $\dot{e}$

$$
\left.F(\chi)=\frac{1+n_{.} H_{\mathrm{o}}}{1-(1-n)_{p} H_{\mathrm{o}}}\left[s^{\mathrm{r}} 1-n\right) p H_{\mathrm{o}}(\operatorname{src} \chi-1)+\ln \text { ro: } \chi\right]
$$

e $I_{\mathrm{o}}$ ha ancora il significato di $l_{\mathrm{MZ}}(\chi=0) \mathrm{ma}$, diversamente da quello definito nella [13'], vale

$$
I_{\mathrm{o}}=\beta h S_{\propto}\left(\frac{T^{*}}{T_{\mathrm{o}}}\right)^{\mathrm{r}-1} \operatorname{ex},\left\{\frac{1+n p H_{\mathrm{o}}}{1-(1-n) p H_{\mathrm{o}}}\left[(1-n) p H_{\mathrm{o}} \mathrm{s}^{\mathrm{r}--}\right]\right\} \text {. }
$$

Si constata subito che $I_{M Z}(\chi)$ ha un eventuale estremo relativo per quei valori di $\chi$ che soddisfano l'equazione

$$
\left[s^{r}(1-n) p H_{0} s c_{\chi}-1\right] \operatorname{tag} \chi=0
$$

ciò̀ per $\chi_{0}=0^{\circ}$ e $\chi_{0}=\operatorname{arcos}\left[s^{r}(1-n) p H_{0}\right]$. Per $\chi=0^{\circ}$ si ha un valore massimo o minimo di $I_{M Z}$ a seconda che sia

$$
\frac{1+n p H_{\mathrm{o}}}{1-(1-n) p H_{\mathrm{o}}}\left[\mathrm{s}^{\mathrm{r}}(1-n) \cdot H_{\mathrm{o}}-1\right]<0 \text { oppure }>0 \text {. }
$$

La seconda soluzione della [24] ha un significato matematice purché sia $(1-n) p H_{0} s^{r} \leqslant 1$; però, anche in questo caso, come vedremo di qui a poco, il valore di $\chi_{\sharp}$ non ha senso fisico. Si può osservare che, se $s^{r} \leqslant 1$, si può definire un angolo $\chi_{\text {: }}$ tale che sia $\cos \chi_{i}=s^{r}$ e che, per la [9], tale angolo è proprio l'angolo zenitale del Sole in 
corrispondenza del quale la quota alla quale si ha il massimo $I_{\mathrm{Mz}}(\chi)$ è quella $c$ alla quale la temperatura assume il valore $T_{1} ;$ la [22] si scrive, per tale valore di $\chi_{.}$,

$$
F\left(\chi_{1}\right)=\frac{1+n p H_{\mathrm{o}}}{1-(1-n) p H_{\mathrm{o}}}\left[(1-n) p H_{\mathrm{o}}\left(1-s^{-\mathrm{r}}\right)+\ln s^{\mathrm{r}}\right] .
$$

Ora l'angolo $\chi_{1}$ cosi definito deve essere evidentemente il limite superiore di variabilità di $\%$ nella formula [22], cui, per valori $\chi>\chi_{1}$, non corrisponde più un effettivo significato fisico. Pertanto l'eventuale angolo $\psi_{2}$ che soddisfa la [24] ha significato fisico se è $1 \geqslant \cos \psi_{2}=$ $=s^{r}(1-n) p H_{0} \geqslant \cos \chi_{1}=s^{r}$; da qui si deduce la condizione necessaria $(1-n) p H_{0} \geqslant 1$ la quale non è mai soddisfatta se $s^{r} \leqslant 1$ in quan. to quest'ultima disuguaglianza equivale alle condizioni

$$
\begin{aligned}
& r<0 \text { ciò̀ }\left(1-n \mid p I_{0}<1 \text { per } p>0\right. \\
& r>0 \text { ciò̀ ancora }(1-n) p H_{o}<1 \text { per } p<0 .
\end{aligned}
$$

É cosi dimostrato che la [21] ha un solo estremo relativo che si consegue per $\chi=0^{\circ}$. La [25], essendo $s^{\mathrm{r}}<1$ e $(1-n) p H_{0}<1$, ha il segrno di $-\left(1+n p H_{0}\right)$; ciò significa che per $\%=0^{\circ}$ ha un valore massimo se $1+n p H_{0}>0$ come in realtà è sempre se deve valere la [8]; tale condizione equivale alle due

$$
\begin{gathered}
\frac{1}{p H_{\mathrm{o}}}>-n \text { per } p>0 \\
-\frac{1}{p H_{\mathrm{o}}}>n \text { per } p<0
\end{gathered}
$$

la prima delle quali poi, tenendo conto delle [27], diviene ancora più restrittiva ciò $\frac{1}{p H_{\mathrm{o}}}>1-n$.

Per renderci conto dell'andamento delle funzioni $I(z), J(z)$ osserviamo che esse sono continue per $z=c$ cioè $I(c)=J(c)$; dalle [10] e [14] si deduce

$$
\begin{gathered}
I(z)=I(c) \exp \left\{\frac{\alpha}{1-(1-i) p H_{\mathrm{o}}}\left[1-\left(\frac{1+r z}{\psi}\right)\right]\right\}\left(\frac{1+p z}{\psi}\right)^{\mathrm{r}-1} \\
\quad \text { da cui }\left(\frac{d I}{d z}\right)_{z=c}=I(c) \frac{1+n p H_{0}}{\psi}\left(s^{\mathrm{r}} \sec \chi-1\right) .
\end{gathered}
$$


Analogamente dalla [15] risulta

$$
\left(\frac{d J}{d x}\right)_{z=c}=I(c) \frac{\alpha \sec \chi^{--1}}{\psi H_{\mathrm{o}}}=\left(\frac{d I}{d z}\right)_{=c}+\frac{n p}{\psi} I(\mathrm{c}) \text {. }
$$

Si vede subito che in generale si ha, per $z=c$, una discontinuitì delle derivate della $I(z)$ e della $J(z)$. Indiehiamo per brevità con $I^{\prime}$ ' e $J_{\mathrm{c}}^{\prime}$ i valori di tali derivate appunto per $z=c$. Essendo $1+n p H_{0}>0$ si ha:

$$
\begin{aligned}
& l^{\prime}=0 \text { a seconda che sia } s^{\prime \prime} \cos \chi \operatorname{cioes} \chi \equiv \chi_{1}, \\
& J_{\mathrm{c}}^{\prime} \equiv 0 \text { a seconda che } \operatorname{sia} \alpha \cos \chi .
\end{aligned}
$$

Per stabilire l'andamento di $I(z)$ e $J(z)$ consideriamo i tre casi $\chi<\chi_{1}, \chi=\chi_{1}, \quad \chi>\chi_{1}$ ciò, rispettivamente, $s^{r} \sec \chi<1,=1,>1$; osserviamo anche che il rapporto $\frac{J_{\mathrm{c}}^{\prime}}{I_{\mathrm{c}}^{\prime}}$ si scrive $\frac{J_{c}^{\prime}}{I_{\mathrm{c}}^{\prime}}-1+\frac{n_{p} H_{\mathrm{w}}}{\left(1+n_{p} H_{\mathrm{o}}\right)\left(\mathrm{s}^{\mathrm{r}} \sec \chi-1\right)}$.

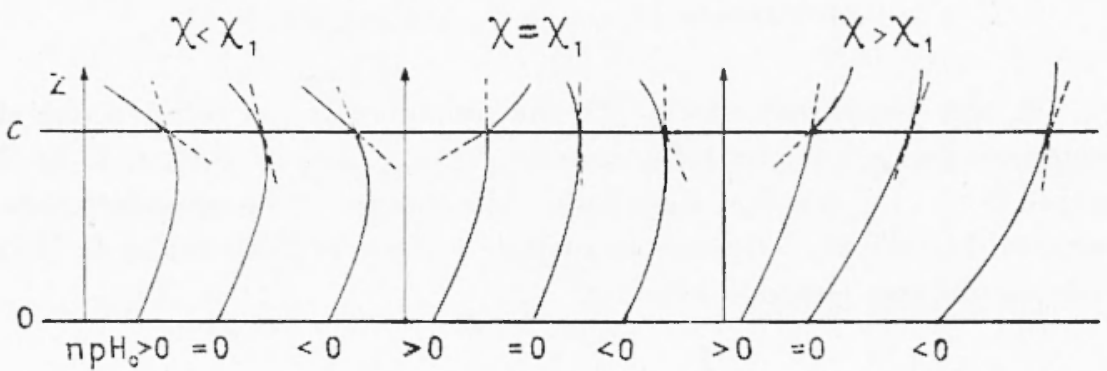

Fig. 1

$1^{\circ}$ caso: $\%<\%$

È sempre $I_{\mathrm{c}}^{\prime}<0$; se inoltre è $n p I_{\mathrm{o}}<0$ si ha senz'altro $J_{\mathrm{c}}<0$ e $\left|J_{\mathrm{c}}^{\prime}\right| \geqslant \mid I_{\mathrm{c}}^{\prime}$ ! mentre se $n p H_{\mathrm{o}}>0$ possono darsi le possibilita $J_{c}^{\prime}=0$ e $\left|J_{c}^{\prime}\right|>$ e $<\operatorname{di}\left|I_{c}^{\prime}\right|$.

$2^{o}$ caso: $\chi=\chi_{1}$

E $I_{\mathrm{c}}^{\prime}=0$ e $\ddot{I}_{n} \equiv \bar{u}$ a scconda che sia $n p \rightleftharpoons 0$.

$3^{\circ}$ caso: $\chi>\chi_{1}$

È sempre $I_{c}^{\prime}>0$; se inoltre è $n p I_{0} \geqslant 0$ è senz'altro $J_{\mathrm{c}}^{\prime}>0 \mathrm{e}$ $\left|J_{\mathrm{c}}^{\prime}\right| \geqslant\left|I_{\mathrm{c}}^{\prime}\right|$ mentre se è $n_{p} H_{\mathrm{o}}<0$ possono aversi le possiluilità $J^{\prime} \cdots 0$ e $\left|J_{\mathrm{c}}^{\prime}\right|>\mathrm{e}<\left|I_{\mathrm{c}}^{\prime}\right|$.

Nella fig. 1 sono indicati andamenti possibili per $I(z)$ e $J(z)$.

In generale il valore del massimo della intensita di ionizzazione (o anche dei massini in quanto possono essercene due, uno al disotto 
e umo al disopra della quola c) e cosi pure le corrispondenti altezze variano con legge assai complessa in dipendenza dei valori attribuiti ai parametri che intervengrono nella teoria.

2. Crso della stratificazione sferica. -- Ci riferiamo in tutto ciò che segue alla fig. 2 . Indichiamo senz'altro con $R$ la distanza tra il centro della Terra $O$ e il livello elıe corrisponde alla quota $z=0$ alla quale la temperatura vale $T_{0}$; il valore di $R$ risulterà nei casi pratici superiore al raggio terrestre di qualche percento. Sia ancora $c$ la quota alla quale la temperatura assume il valore $T_{1} ; Q Q^{\prime} Q^{\prime \prime}$ la direzione di incidenza

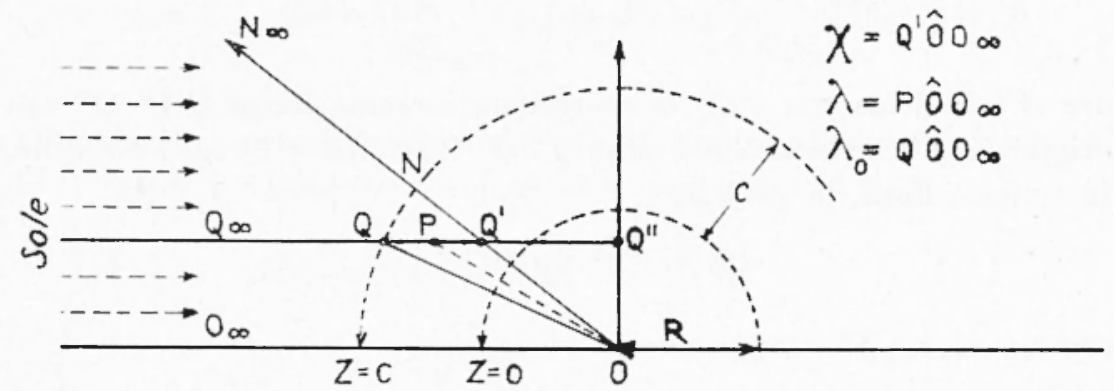

Fig. 2

di un raggio della radiazione ionizzante proveniente dal Sole e sia $Q^{\prime}$ il punto in cui tale raggio interseca la direzione $O N$ (asse $z$, corrispondente ad un angolo zenitale $\%$ ) lungo la quale vogliamo determinare landamento della intensita di ionizzazione; indichiamo inoltre con $\zeta$ la grenerica coordinata $z$ che corrisponde al punto $P$ del segmento $Q Q^{\prime}$ lungo la direzione $O P$ formante un angolo $\lambda$ con la direzione della radiazione proveniente dal Sole; l'angolo $\lambda$ varia tra i limiti $\lambda_{0}$, inferiore, e $\chi$, superiore, per i punti del segmento $Q Q^{\prime}, 0$ e $\lambda_{0}$ per i punti della semiretta $Q Q_{\alpha}$; quando poi considereremo la intensità di ionizzazione lungo la semiretta $N N$ invece dovremo far variare l'angolo $\lambda$ tra 0 e $\%$.

La densità di materia lungo l'asse $Q Q^{\prime}$ si scrive

$$
\rho=p_{\mathrm{o}} \exp \left[-\frac{1}{H_{\mathrm{n}}} \int_{\mathrm{o}}^{\zeta}\left(1+H_{\mathrm{o}} \frac{d f}{d z}\right) \frac{d z}{f(z)}\right]
$$

con $\rho_{0}=\rho(0)$ come nella $[2]$. 
Per la direzione $Q Q^{\prime} Q^{\prime \prime}$ si ha

$$
O Q^{\prime \prime}=q=(R+z) \operatorname{sen} \chi=(R+\zeta) \operatorname{sen} \lambda
$$

da cui

$$
\lambda(\zeta)=\operatorname{arsen}\left[\frac{R+z}{R+\zeta} \operatorname{sen} \chi\right]
$$

La relazione che dà l'assorbimento, analogamente al caso della [3], si può scrivere

$$
\frac{d S}{S}=\frac{A_{\mathrm{o}}}{[f(\zeta)]^{\mathrm{n}-1}} p_{\mathrm{o}} \exp \left[-\frac{1}{H_{\mathrm{o}}} \int^{\zeta}\left(1+H_{0} \frac{d f}{d z}\right) \frac{t z}{f(z)}\right] d x(\zeta)
$$

ove si è indicata con $x(\zeta)$ la coordinata corrente lungo $Q Q^{\prime} Q^{\prime \prime}$ con origine in $Q^{\prime \prime}$ e orientata nel senso $Q^{\prime \prime} Q^{\prime}$. E $x=(R+\zeta) \cos \lambda$ cosicché, in termini finiti, la [36] dà

$$
S(\zeta)=S^{*}, x p[h G(\zeta)]
$$

$\operatorname{con} h=A_{\mathrm{o}} \rho_{\mathrm{o}}, S^{*}=S(c)$ come in $[4]$ e con

$$
G(\zeta)=\int_{c}^{\zeta} t(z, \xi)+x p\left[-\frac{1}{H_{0}} \int_{0}^{\xi}\left(1+H_{\circ} \frac{d f}{d z}\right) \frac{d z}{f(\Sigma)}\right] \cdot[f(\xi)]^{-\mathrm{n}+1} d \xi
$$

ove si è introdotta la funzione $t(z, \xi)=\left[1-\left(\frac{R+z}{R+\xi}\right)^{2} \operatorname{sen}^{2} \chi\right]^{-1 / z}$ delle
variabili $z$ e $\xi$.

La intensità di ionizzazione $I \zeta$ i lungo $Q Q^{\prime} Q^{\prime \prime}$ vale

$I(\zeta)=\beta h \supset^{*} \exp \left[h G(\zeta)-\frac{1}{H_{\mathrm{o}}} \int_{\mathrm{o}}^{\zeta}\left(1+H_{\mathrm{o}} \frac{d f}{d z}\right) \frac{d z}{f(z)}\right] \cdot[f(\zeta)]^{-n-1-1}$

la quale, alla quota $z$ corrispondente a $Q^{\prime}$ lungo la direzione $O N$, diviene

$$
\begin{gathered}
I(z)=\beta h S^{*}, x p[\Phi(z)] \cdot[f(z)]^{-\mathrm{n}+1} \\
\operatorname{con} \Phi(z)=h G(z)-\frac{1}{H} \int_{0}^{\mathrm{z}}\left(1+H_{\mathrm{o}} \frac{d f}{d z}\right) \frac{d z}{f(z)}
\end{gathered}
$$



$\frac{d I}{d z}=0$

Eventuali punti di estremo relativo di $I(z)$ si possono avere per

Questa volta occorre però notare che, a differenza del caso di stratificazione piana, nella derivazione di $I(z)$ rispetto a $z$ va considerata la dipendenza di $S^{*}$ oltre che da $\not$ come nella [4] anche dalla stessa variabile $z$; notiamo ancora che nella [40] la $G(z)$ è funzione di $z$ sia nel limite superiore dell'integrale sia, anche, nello stesso integrando.

La dipendenza di $S$ da $z$ e da $\chi$ per $\zeta \geqslant c$ è espressa, avendo posto $f(z)=1+p z$, dalla formula

$$
S(\zeta ; z, \chi)=S_{\infty} \exp \left[-\frac{\alpha}{\zeta H_{0}} \int_{\zeta}^{\infty} t(z, \xi) \exp \left[-\frac{\zeta-c}{\zeta H_{\mathrm{o}}}\right] d \xi\right] .
$$

Da questa espressione ponendo $=c$ il limite inferiore dell'integrale si ricava il valore $S^{*}$ e quindi, in conseguenza, si ha

$$
I(z)=I(c) \exp \left[\tau(z) \cdot\left(\frac{1+p z}{\psi}\right)^{r-1}\right.
$$

$\operatorname{con} I(c)=\beta \frac{\alpha S_{x}}{\psi H_{0}} \exp \left[-\frac{\alpha}{\psi H_{0}} \int_{c}^{\infty} \operatorname{cxp}\left(-\frac{\xi-c}{\psi H_{0}}\right) \cdot t(c, \xi) d \xi\right]$

$$
\mathrm{e} \tau(z)=h G(z)-\frac{\alpha}{\psi H_{\circ}} \int_{\mathrm{c}}^{\infty} \exp \left(-\frac{\xi-c}{\psi H_{\circ}}\right)[t(z, \xi)-t(c, \xi)] d \xi
$$

La equazione $\frac{d I}{d z}=0$ si traduce allora nell'altra

$$
h \sec \gamma(1+p z)^{r}-\left(n p+\frac{1}{H_{0}}\right)=(1+p z)\left[-h B(z)+\frac{\alpha}{H_{0}}(:(z)]\right.
$$

ove si è posto

$$
\begin{gathered}
B(z)=(R+z) s n^{2} \% \int_{c}^{\mathrm{z}} \frac{(1+p \xi)^{\mathrm{r}-1}}{(R+\xi)^{2}}[t(z, \xi)]^{3} d \xi \\
C(z)=(R+z) \operatorname{sen} \% \int_{\mathrm{c}}^{\infty} \exp \left[-\frac{\xi-c}{\psi H_{\mathrm{o}}}\right] \frac{\left[t(z, \xi,]^{3}\right.}{(R+\xi)^{2}} d \xi
\end{gathered}
$$


La equazione [44] si identica con la [8] nel caso $\chi=0^{\circ}$ e quindi, anche nel caso di stratificazione sferica, vale la $\left[9^{\prime}\right]$.

Vogliamo ora considerare la $J(z)$, da definire come nel paragr. 1 per $z>c$; si ha

$$
\begin{aligned}
& J(z)=J(c) \exp \left|-\frac{\alpha}{\psi I_{0}} \sigma(\xi)-\frac{z-c}{\psi H_{0}}\right| \\
& \operatorname{con} J(c)=\beta \frac{\simeq S_{-}}{\psi H_{0}} \exp \left[-\frac{\alpha}{\psi H_{0}} \int_{\varepsilon}^{\infty} t(c, \xi) \exp \left[-\frac{\xi-c}{\psi H_{0}}\right] d \xi\right] \\
& \text { e } \sigma(z)=\int_{\mathrm{z}}^{\infty} t(z, \xi) \exp \left[-\frac{\xi-c}{\psi H_{\mathrm{o}}}\right] d \xi-\int_{\mathrm{c}}^{\infty} t(c, \xi) \exp \left[-\frac{\xi-r}{\psi H_{0}}\right] d \xi
\end{aligned}
$$

Un eventuale massimo relativo di $J(z)$ si ha per $\frac{d J}{d z}=0$ che equivale a

$\alpha \sec \chi \exp \left[-\frac{z-c}{\psi H_{\mathrm{o}}}\right]-1=\alpha(R+z) \operatorname{sen}^{2} \times \int_{\mathrm{z}}^{\infty} \exp \left[-\frac{\xi-c}{\psi H_{\mathrm{o}}}\right] \frac{[t(z, \xi)]^{3}}{(R+\xi)^{2}} d \xi$.

Si verifica immediatamente dalle [43] e [48] l"identità $I(c)=J(c)$. In quanto alle derivate $I_{\mathrm{c}}^{\prime}$ e $J^{\prime}$, calcolate alla quota $c$, si ha

$$
\begin{gathered}
I_{\mathrm{c}}^{\prime}=I(c) \frac{\omega \cdots \alpha-1-n p H_{\mathrm{o}}-\alpha C(c)}{\psi H_{\mathrm{o}}} \\
J_{\mathrm{c}}^{\prime}=J(c) \frac{\alpha \sec \chi-1-\alpha C(c)}{\psi H_{\mathrm{o}}}=I_{\mathrm{c}}+\frac{{ }^{n} p}{\psi} I(c)
\end{gathered}
$$

Osserviamo infine che il massimo della intensita di ionizzazione $I(z)$ viene a trovarsi alla quota $c$ quando

$$
s^{\mathrm{r}}=\frac{1}{\sec \%-C(c)}
$$

che, ricordando la posizione $s^{\mathrm{r}}=\cos \psi_{1}$, diviene

$$
\sec \chi=\sec \chi_{1}+C(c) \text {. }
$$


Si constata nelle [51], [52] la identita formale con le corrispondenti [30], [31] pur di sostituire in queste ultime

$$
\sec \% \operatorname{con} \sec \%-C(c) \text {. }
$$

3) Confronto e discussione dei risultati ottenuti. - Si verifica immedialamente che per $R \rightarrow \infty$ le formule ottenute nel paragrafo 2 per le $I(z), J(z), I_{c}^{\prime}, J_{c}^{\prime}$, ecc. si identificano con le corrispondenti ottenute nel paragrafo 1.

Per stabilire i limiti di validita della teoria approssimata sviluppata nel paragrafo ] occorre esaminare le [10] e [42], le [15] e [47], le $[8]$ e $[44]$, le $[16 \mid$ e $[50]$.

La $I(z)$ espressa dalla [42], per il primo teorema della media come si constata con facilità, soddisfa alle limitazioni

$$
\begin{aligned}
f(z) & >\beta h S_{\infty} \exp [h \sec \% g(z)-\alpha \sec \%] \cdot(l+p z)^{r-1} \\
& \left.<\beta h S_{\infty} \exp \mid h g(z) t(z, c)-\alpha\right](1+p z)^{r-1}
\end{aligned}
$$

ove $g(z)$ è quella definita nella [4] e ove si sono effettuate le integrazioni che, appunto applicando il teorema della media, sono divenute possibili per via elementare. Il membro di destra della prima delle [55] c proprio la $I(z)$ nel caso di stratificazione piana e possiamo pertanto dire che la $I(z)$ espressa dalla $[42]$ risulta ad ogni quota magriore della corrispondente [29]: cioè l'assorbimento al disopra di una qual-iasi quota è minore nel caso di stratificazione sferica che in quello di stratificazione piana.

Un risultato analogo si stabilisce per la $J(z)$ è precisamente

$$
\begin{aligned}
J(z) & >\beta \frac{\alpha S_{\infty}}{\psi H_{0}} \operatorname{ex!}\left[-\alpha \sec \gamma \exp \left(-\frac{z-c}{\psi H_{0}}\right)-\frac{z-c}{\psi H_{\mathrm{o}}}\right] \\
& \left.<\beta \frac{\alpha S_{\infty}}{\psi H_{o}} \operatorname{xp} \mid-\alpha c x p\left(-\frac{z-c}{\psi H_{0}}\right)-\frac{z-c}{\psi H_{\mathrm{o}}}\right]
\end{aligned}
$$

ove ancora il termine di destra della prima delle [56] coincide con la [15] del paragrafo 1.

In quanto all'altezza del massimo di intensità di ionizzazione si vede che il primo membro della [44] coincide con il primo membro della $[8]$; si constata immediatamente che, per essere $C(z)$ e -- $B(z)$ 
quantitì positive, il valore di $z$ che soddisfa la [44] è inferiore a quello che soddisfa la [8]: in altri termini l'innalzamento della quota alla quale si registra un massimo di $l(z)$ al crescere di $\%$ è, nel caso di stratificazione sferica, meno sensibile che nel caso di stratificazione piana; questo risultato è mostrato in diversa forma dalla [54] secondo cui il valore di $\%$ per il quale il massimo si trova alla quota $c$ risulta magroriore che nel caso di stratificazione piana.

Analogamente per la $J(z)$ si nota che il primo membro della [50] coincide con il primo membro della [16] e anche in questo caso é immediato constatare che la presenza del termine positivo a secondo membro della [50] equivale ad una diminuzione del valore di z che soddisfa la [50] stessa.

In quanto all'andamento delle funzioni $I(z)$ e $J(z)$ si osserva l'identitì formale delle $\mid 3]$ e [52] e l'uguaglianza della differenza $\frac{J_{\mathrm{c}}^{\prime}}{J(c)}-\frac{I_{\mathrm{c}}^{\prime}}{I()}=\frac{J_{\mathrm{c}}^{\prime}-I_{\mathrm{c}}^{\prime}}{I(c)}=\frac{n p}{\bigcup}$ nei due casi di stratificazione piana e sferica; in quest'ultimo caso però la $l(c)$ risulta numericamente magrgiore che in quello cosicché il salto di derivata $J_{c}^{\prime}-I^{\prime}{ }_{c}$ risulta superiore che nel caso trattato nel paragrafo 1 . Se ne conclude che l'andamento della intensiti di ionizzazione risulta di tipo essenzialmente analogro a quello rappresentato nella figr. 1 pur di sostituire le tre condizioni $\chi<\%_{1}, \%=\%_{1} \%>\%_{1}$ con ie altre tre condizioni $\%<\%_{i 3}$, $\chi=\chi_{33}, \chi>\chi_{3}$ essendo $\chi_{i 3}>\chi_{1}$ l'angolo $\chi$ che soddisfa la [54].

A differenza della teoria originaria di Chapman il margior numero dei parametri contenuti nella presente teoria rende più complesso lo stabilire i limiti entro cui la trattazione esatta del paragrafo 2 può essere sostituita senza sensibile errore dalla teoria approssimata del paragrafo 1: dai primi risultati numerici elaborati sembra comunque che tale approssimazione valga almeno per angoli zenitali inferiori a $60^{\circ}$.

Roma - Istituto Nazionale di Geofisica - Luglio $195 \%$.

\section{RIASSUNTO}

Si sviluppa la teoria della fotoionizzazione di Chapman nel caso di atmosfera non isoterma; si considera in particolare un'atmosfera nella quale la temperatura varia linearmente con lalteza fino ad 
una certa quota e quindi assume un valore costante. I risultati ottenuti per l'intensità di ionizzazione nei due casi in cui l'atmosfera è supposta stratificata secondo piani paralleli (raggio della Terra $=\infty$ ) oppure secondo superficie sferiche concentriche alla Terra (raggio di questa finito) coincidono praticamente almeno per angoli zenitali non superiori a 60". Differenze sensibili si riscontrano nella forma della dipendenza dell'intensità di ionizzazione e dell'altezza del suo massimo (o dei suoi massimi) rispetto alla teoria originaria di Chapman.

\section{$S U M M A R Y$}

IF' state the theory of the Chapman photoionization in a non isotherm atmosphere; we particularly consider an atmosphere in which the temperature varies linearly with the height at least up to a certain altitude and thus assumes a constant value. The results obtained for the ionization intensity in the two cases in which the atmosphere is supposed stratified in parallel planes (radius of the earth $=\infty$ ) or spheres concentric to the earth are pratically coincident at least for zenith angles less than $60^{\circ}$. Notable differences are found in the form of the dependence of the ionization intensity and of the height of its maximum (or maxima) with respect to the original Chapman theory.

\section{BIBLIOGRAFIA}

(1) Chapuin S., Ploc. Phys. Soc. 1931, 43, 26.

(2) Chapman S., Proc. Phys. Soc. 1931, 43, 483.

(3) Gerson N. C., Rep. Progr. Pliys. 1951, 14, 316.

(4) Gezbhill J. A. Szendrei M. E. Proc. Phys. Soc. B, 1950, 63, 427.

(j) Bates D. R., Proc. Phys. Soc. B, 1951, 64, 805. 